\title{
T-cell therapy targeting stromal cells may improve treatment of solid tumors
}

The layer of cells surrounding solid tumors, termed the stroma, may be a new therapeutic target, say researchers at the University of Chicago (IL, USA). "We al ready knew that targeting the stroma is essential for eradicating established large tumors, because the stroma is like the 'root' of the tumor", said $H$ ans Schreiber, author of the study.

$M$ any solid tumors become resistant to chemotherapy or treatments that boost the response of the immune system to the tumor because the genetic instability of the tumor cells results in the antigenpresenting cells being discarded. Consequently, white blood cells do not kill the cancerous cells because they do not recognize them as harmful. The tumors then grow rapidly.

“...the stroma is like the 'root' of the tumor"

H owever, it has been shown that tumor antigens can accumulate in the stroma. U nlike tumor cells, the cells of the stroma are gen etically stableand have retained the antigen-presenting cells. Presenting tumor antigens on their surface makes the stromal cells a good target for immunotherapy.

Explaining the reasoning behind the current study, Schreiber said, "Since cancer is a genetic disease, we wondered whether mutant proteins are released into the surroundings and picked up by the stroma. If so, we can target the root of the cancer in a cancer-specific way to arrest or eradicate a tumor".

The researchers injected $T$ cells that were specifically engineered to recognize the tumor antigen into mice with large established cancers. The T cells killed the stromal cells but did not have a direct impact on the cancerous cells. As a result, the tumor size was reduced and growth was inhibited for more than 80 days. The researchers noted that not all cancer cells were eliminated, but that targeting the stroma with a single injection of $T$ cells did stop or slow the growth of well-established cancers. "Such growth arrest in patients", said the authors, "would be an admirable achievement for many cancers and could also be used as an adjuvant to other therapies".

\section{"Only tumor-derived stromal cells appear to pick up and present tumor antigen"}

O bviously tumor eradication is preferable to arresting tumor growth; however, as coauthor Bin Zhang (University of Texas $\mathrm{H}$ ealth Sciences C enter, TX, U SA) says, the results of this study are significant because “we can't target cancer cells when they have lost their antigen-presenting molecules". Therefore, "... stroma becomes an ideal al ternative target for T cells".

Importantly, the researchers did not find evidence of healthy cells in other tissues becoming targets by picking up the antigens, which would be a major concern. "O nly tumor-derived stromal cells appear to pick up and present tumor antigen", said Zhang.

“...targeting the stroma with a single injection of Tcells stopped or slowed the growth of well-esta blished cancers."

Further research of this therapeutic approach is required. The next step will involve testing $T$-cell therapy in melanoma, colon and breast cancer. "We know that stromal cells often present tumor-antigen in these tumors, but have not yet performed $\mathrm{T}$-cell therapy on these mice", said Z hang. Furthermore, early results from studies of this approach in human cancers suggest that it “... might be useful for the human situation as well".

Source: Zhang B, Zhang Y, Bowerman NA et al.: Equilibrium between host and cancer caused by effector T cells killing tumor stroma. Cancer Res. 68(5), 1563-1571 (2008). 


\section{Priority Paper Alerts}

Rad9 has a functional role in human

prostate carcinogenesis.

Zhu A, Zhang CX, Lieberman HB: Cancer Res. 68(5),

1267-1274 (2008).

This study aimed to determine the relationship between the cell-cycle checkpoint protein Rad9 and prostate cancer. At present, prostate cancer is the second leading cause of cancer death in males. Four prostate cancer cell lines were found to have aberrantly high Rad9 levels compared with PrEC normal prostate cells. It was demonstrated that the elevated Rad 9 levels in prostate cancer cells are due, at least in part, to hypermethylation or amplified Rad9 copy number. siRNA was utilized to lower the levels of Rad9 protein in the prostate cancer cell lines and this correlated with reduced tumorigenicity in nude mice. This indicates that Rad9 actively contributes to the disease. Human biopsy samples were also examined and Rad9 levels were found to be elevated in 153 of 339 human prostate tumor samples, but were only detectable in two of 52 noncancerous prostate tissues. Rad9 level was also associated with cancer stage and it may therefore be utilized as a biomarker and therapeutic target for advanced prostate cancer.

Targeting vascular endothelial growth factor in advanced carcinoid tumor: a random assignment Phase II study of depot octreotide with bevacizumab and pegylated interferon $\alpha-2 b$.

Yao JC, Phan A, Hoff PM et al.: J. Clin. Oncol. Carcinoid tumors are tumors of the neuroendocrine system. Currently, there is a lack of an effective systemic therapy for advanced carcinoid. This study aimed to investigate the combination of bevacizumab (BEV) and pegylated (PEG) interferon $\alpha-2 b$ in patients with metastatic or unresectable carcinoid tumors. A total of 44 patients were randomly assigned to receive either BEV or PEG interferon $\alpha-2 b$ for 18 weeks. At disease progression or after 18 weeks (whichever was sooner), patients received BEV plus PEG interferon until progression. It was found that at 18 weeks, $18 \%$ achieved confirmed partial response, $77 \%$ had stable disease and $5 \%$ had disease progression in the BEV arm. By comparison, $68 \%$ had stable disease and $27 \%$ had disease progression in the PEG interferon arm. After 18 weeks of monotherapy, progression-free survival rates were 95 and $68 \%$ in the BEV and PEG interferon arms, respectively. A significant decrease in tumor blood flow was observed in patients treated with BEV, and a significant decrease in plasma basic FGF and an increase in plasma IL-18 were observed in PEG interferon-treated patients. The study concludes that BEV therapy resulted in reduced tumor blood flow and longer progressionfree survival in patients with carcinoid tumors compared with PEG interferon treatment.

\section{Progress towards completing the picture of colorectal cancer genetics}

$\mathrm{NIH}$-funded research reported

in the $M$ arch issue of the American Journal of H uman Genetics identifies new genes thought to be implicated in colorectal cancer (CRC).

O nly $5 \%$ of $C R C$ cases are considered to be the product of aberrant genes; however, it is likely that there is a large number of genes with a role in CRC that are yet to be identified. This is the result of the complicated genetics governing the changes associated with CRC: the genes so far identified have strong links to $C R C$, while others that remain elusive likely have small roles individually, but combine to lead to CRC.

'... it is likely that there is a large number of genes with a role in CRC that are yet to be identified'

Completing our knowledge of the genetics of CRC would improve diagnostics and improve patient outcome. $\mathrm{H}$ ereditary CRC typically develops prior to the age at which national screening programs take effect. 0 ffering full genetic screening for CRC would enable physicians to take full advantage of more intensive diagnostics, such as colonoscopy, at an earlier stage in the most at-risk patients.
This latest study, a genomewide scan with linkage analysis in 194 families with a history of CRC, was designed to identify other genes that likely contribute to CRC. The researchers homed in on chromosome 9, to a region that has a known CRC-susceptibility gene. From the resulting linkage analysis, the researchers were able to pinpoint additional $C R C$-susceptibility regions on chromosomes $1 p, 15 q$ and $17 p$.

'Researchers were able to pinpoint CRC-susceptibility regions on chromosomes $1 p, 15 q$ and 17p'

The C ase-Western researchers also investigated cancer clusters, such as CRC and breast cancer and $C R C$ and multiple polyps, in families in an effort to examine the possibility that multiple susceptibility genes cause different types of cancer. Georgia $L$ Weisner, an author of the study, commented, "the goal of our study is to identify the CRC genes in susceptible patients to better understand who may be prone to develop CRC and why".

Source: D aley D, Lewis S, Platzer $P$ et al.: Identification of susceptibility genes for cancer in a genome-wide scan: results from the colon neoplasia sibling study. Am. J. H uman Genet. 82(3), 723-736 (2008).

\section{About the Bulletin Board}

The Bulletin Board highlights some of the most important events and research in the field of oncology.

If you have newsworthy information, please contact: Victoria Rawley, Commissioning Editor, Future Oncology; Future Medicine Ltd, Unitec House, 2 Albert Place, London N3 1QB, UK

Tel.: +44 (0)20 8371 6090; Fax: +44 (0)20 83432313

v.rawley@futuremedicine.com 


\section{Explaining the reason why $\mathrm{H}$ erceptin ${ }^{\circledast}$ is not effective in all cases}

The popular breast cancer drug

H erceptin ${ }^{\circledR}$ does not work in up to half of women taking it, and a likely reason for this has been unearthed by researchers at the $U$ niversity of $C$ alifornia $D$ avis $C$ ancer Center (CA, USA).

When it works, $\mathrm{H}$ erceptin, which is taken by approximately $25 \%$ of women with breast cancer, decreases the risk of relapse and extends patient survival.

According to Colleen Sweeny, senior author of the study, "H erceptin revolutionized the treatment of breast cancer, but our clinical experiences indicate that there is room for improvement. We wanted to find out what was reducing $\mathrm{H}$ erceptin's effectiveness in some cases."

H erceptin, introduced in 1998, is used to treat women who have tested positive for multiple copies of the Her2 gene, which is responsible for aiding cancer cell growth and spread. $\mathrm{H}$ erceptin works by blocking the $\mathrm{H}$ er2 cell-growth signal; this results in a slowing of the cancer cell growth.

The team of researchers had previously demonstrated the presence of another gene called $M$ et that, if activated at the same time as $\mathrm{H}$ er2, makes tumor cells more invasive. W ith this knowledge of the $M$ et gene, the team conducted a series of experiments to assess whether $M$ et also contributed to H erceptin resistance. They found that the combination of $\mathrm{M} \in$ and $\mathrm{H}$ er2 activation significantly increased tumor-cell proliferation, and theactivation of $M$ et reduces $H$ erceptin's effectiveness.

Sweeny explains: "T he M et gene is waiting in the wings and can take over from $\mathrm{H}$ er2 when it is targeted with $\mathrm{H}$ erceptin. Some H er2-positive tumor cells express $M$ et and these cells respond to $\mathrm{H}$ erceptin by making even more copies of $M$ et. When a woman gets breast cancer, we currently test her tumor for $\mathrm{H}$ er 2 but don't take $M$ et into account. We believe that looking at just $\mathrm{H}$ er2 status is no longer going to tell us the whole story."

The team is now planning to determine whether $M$ et expression predicts $\mathrm{H}$ erceptin resistance. "W e need to confirm that the $M$ et receptor is going to be a viable target. If we again find a significant correlation between $M$ et and $\mathrm{H}$ erceptin resistance, the next step will be drug studies targeting $M$ et in an animal model," Sweeny concludes.

Source: Shattuck DL, M iller JK, Carraway KL 3rd, Sweeney C: $M$ et receptor contributes to trastuzumab resistance of $\mathrm{H}$ er2-overexpressing breast cancer cells. Cancer Res, 68(5), 1471-1477 (2008).

\section{Cancer screening guidelines may neglect danger signs}

The American Cancer Society has updated its colon cancer screening guidelines to include two novel techniques, despite concerns that one of the methods may not detect an al ready underidentified indicator of cancer risk.

V irtual colonoscopy uses a CT scan to identify precancerous polyps, which can often develop into tumors. This technique is hoped to be useful because it does not require the use of a rectal probe, which can put many people off screening.

$\mathrm{H}$ owever, a study published in the $M$ arch issue of the Journal of the American $M$ edical Association raises concerns that the technique may overlook an underrecognized indicator of colon caner. N onpolypoid colorectal neoplasms (N P-CRN s) are flat or depressed, precancerous lesions that were previously only identified as risk factors in Japanese studies. The study, by researchers at the Veterans Affairs Palo Alto $\mathrm{H}$ ealth $\mathrm{C}$ are System (CA, USA), found that such lesions are also common in Americans and, the authors suggest, are frequently overlooked by doctors performing colonoscopies. In total, $9.35 \%$ of the 1819 military veterans examined had N PCRN s. W hile flat or depressed lesions accounted for only $15 \%$ of the potentially cancerous growths found in the study, they were associated with almost $50 \%$ of subsequent cancers, suggesting that they are far more dangerous than traditional polyps. As they do not stick out and appear relatively similar to healthy tissue, it is thought that many doctors, especially those who rush colonoscopies, miss them. Virtual colonoscopy, which can only distinguish abnormal growths in the colon, is almost certainly unable to pick up N P-CRN s.

D ouglas Rex of Indiana U niversity (IN , U SA) feels doctors need further training and practice to ensure N P-CRN S are identified. "I think there are a lot of people who expect everything in there to be shaped like a golf ball. It's not." In response to such claims, Roy Soetikno, first author on the study, has started an exchange program in the hope that American doctors will learn to identify flat lesions from their experienced Japanese counterparts.

D espitethe shortcomings in the virtual colonoscopy approach, many feel that because it is a less invasive procedure and is therefore less off-putting, introduction of the technique is vital to increase the coverage of colorectal cancer screening, even if N P-CRN s are missed. Grace Elta, of the University of M ichigan (MI, USA), explains: "Fifty percent of the (US) population gets no screening. Any screening is better than that."

Sources:

www.nytimes.com/2008/03/06/health/research/0 6 cancer.html?ref=health

www.nytimes.com/2008/03/05/health/research/0 5 cancer.html? ref=research

Soetikno RM , Kaltenbach T, Rouse RV et al.: Prevalence of nonpolypoid (flat and depressed) colorectal neoplasms in asymptomatic and symptomatic adults. JAM A 299(9), 1027-1035 (2008). 